SHORT REPORT

\title{
Creutzfeldt-Jakob disease 38 years after diagnostic use of human growth hormone
}

\section{E A Croes, G Roks, G H Jansen, P C G Nijssen, C M van Duijn}

A 47 year old man is described who developed pathology proven Creutzfeldt-Jakob disease (CJD) 38 years after receiving a low dose of human derived growth hormone (hGH) as part of a diagnostic procedure. The patient presented with a cerebellar syndrome, which is compatible with iatrogenic CJD. This is the longest incubation period described so far for iatrogenic CJD. Furthermore, this is the first report of CJD after diagnostic use of $h \mathrm{GH}$. Since the patient was one of the first in the world to receive hGH, other cases of iatrogenic CJD can be expected in the coming years.

$\mathrm{P}$ rion diseases are potentially transmissible. Human to human transmission was first reported in 1974, when a 55 year old woman was described who developed symptoms of Creutzfeldt-Jakob disease (CJD) 18 months after a corneal transplant. ${ }^{1}$ Since then, transmission has been reported after stereotactic electroencephalographic (EEG) depth recording, human growth hormone (hGH) and gonadotrophin treatment, and dura mater transplantation. ${ }^{2-5}$ More than 267 patients with iatrogenic CJD are known today and their number is growing. ${ }^{6}$ The most important iatrogenic cause of CJD is still contaminated cadaveric hGH. Exposure to contaminated hGH occurred before 1985, when recombinant growth hormone became available. In a recent study, incubation periods in 139 patients with hGH associated CJD were found to range from 5-30 years, with a median of 12 years. ${ }^{6}$ One of the factors influencing incubation time is genotype on polymorphic codon 129 of the prion protein gene. ${ }^{7}$ The incubation time is significantly shorter in people who are homozygous for either methionine or valine on this polymorphism. ${ }^{7}$

We describe the second patient with hGH related CJD in the Netherlands. The patient developed the disease 38 years after hGH injections. To our knowledge, this is the longest incubation period described for any form of iatrogenic CJD. Furthermore, our patient was not treated with hGH but only received a low dose as part of a diagnostic procedure.

\section{CASE REPORT}

This patient presented at the age of 47 years with paraesthesia in both arms for six months, difficulty with walking for four weeks, and involuntary movements of mainly the upper extremities of two weeks' duration. He did not notice any change in cognitive function, although his twin sister had noticed minor memory disturbances. There was no family history of neurological disease. During childhood the patient had experienced a growth delay compared with his twin sister and with the average in the Netherlands. When he was 9 years old, a nitrogen retention test with $6 \mathrm{IU}$ hGH over five days was performed to exclude growth hormone deficiency. Since the result was not decisive, a quantitative amino acid test was performed, which measures 30 amino acids during fasting and one, two, and three hours after growth hormone injection. No abnormal amino acid concentrations were found making the diagnosis of primordial dwarfism most likely. Therefore, no treatment with hGH was given.

On neurological examination we found a slight dysarthria without aphasia. Cranial nerve function was normal. Walking was unstable and wide based. During movements of the upper extremities myoclonic jerks were present. Sensation, muscle tone, and strength were normal. Co-ordination was impaired in all four limbs with a disturbed balance. Tendon reflexes were brisk at the arms and increased at the legs with a clonus in the ankle reflex. Plantar responses were both normal. On the mini mental state examination, the patient scored 30/30. Routine laboratory investigation, thyroid function, vitamin concentrations (B-1, B-6, B-12, and E), and copper metabolism were normal. Admission EEG examination showed generalised arrhythmic slow activity with diffuse spikes and spike waves. EEG examination two months later showed a further slowing of the rhythm with bilateral diphasic sharp waves but was not typical for CJD. Cerebral magnetic resonance imaging was normal. Cerebrospinal fluid examination showed l cell $/ 3 \mu \mathrm{l}$, normal glucose and protein concentrations, and a strongly positive $14-3-3$ protein test. The patient was homozygous for methionine on the PRNP codon 129 polymorphism. On clinical grounds, CJD was diagnosed.

Within one month the patient's condition deteriorated rapidly and because of severe disturbances in coordination and progressive myoclonus he became bedridden. An eye movement disorder developed with slow saccadic and dysmetric eye movements. Temperature became unstable with peaks of $39^{\circ} \mathrm{C}$ without an infectious focus, for which a disorder of autoregulation was presumed. Until a very advanced stage, cognitive function was intact. The patient died five months after admission. The diagnosis of CJD was confirmed at necropsy. The brain weighed $990 \mathrm{~g}$ and showed clear cortical and cerebellar atrophy. Spongiosis, neuronal loss, and gliosis were found predominantly in the putamen, caudate nucleus, and basotemporal and cerebellar cortex; the cerebellum was the most severely affected of these. Vacuoles ranged from 2-12 $\mu \mathrm{m}$. No amyloid or Kuru plaques were found. Immunohistochemical staining (3F4 antibody 1:1000, Senetek, USA) was clearly positive for prion protein accumulation in a "synaptic" distribution. Most deposition was found in the stratum moleculare of the cerebellum.

\section{DISCUSSION}

We describe a 47 year old patient who developed pathology proven CJD 38 years after hGH injections. The patient was never treated with hGH but received a small dose as part of a

Abbreviations: CJD, Creutzfeldt-Jakob disease; EEG, electroencephalographic; hGH, human growth hormone 
diagnostic procedure. The onset of CJD was signalled by prodromal symptoms of paraesthesia followed by a rapidly progressive ataxia. The disease presentation and course with predominantly cerebellar and eye movement disorders are compatible with iatrogenic CJD caused by hGH treatment. ${ }^{68}$

Growth hormone treatment was first described in 1958 but hGH was not produced on a larger scale from human pituitary glands until the beginning of the 1960s. In the Netherlands growth hormone extraction started in 1963 and was soon centrally coordinated. Until 1979 growth hormone was extracted non-commercially from pituitaries by a pharmaceutical company. In 1971 commercial products also became available. Our patient was one of the first to receive hGH in the Netherlands but the origin of this product was not recorded. A causal relation can therefore not be established with full certainty, but coincidentally receiving growth hormone and developing this very rare disease is unlikely. Since the clinical course in this relatively young patient is in accordance with an iatrogenic cause, we think the probability is high that the hGH injections explain the development of CJD in this patient.

The first Dutch patient with hGH related CJD died in 1990. ${ }^{9}$ During several periods from 1963 to 1969 she received intramuscular injections of hGH. During an unknown period the hGH was derived from South America. At age 39, 27 years after starting the treatment, she developed an ataxic gait, slurred speech, sensory disorders, and myoclonus, but her cognitive function remained normal. Postmortem examination of the brain confirmed the diagnosis of CJD. ${ }^{9}$ Following the identification of this patient, a retrospective study was started to trace all 564 registered hGH recipients who were treated before May 1985. Until January 1995, none of these was suspected of having CJD. ${ }^{10}$ Since 1993 prospective surveillance for all forms of human prion disease has been carried out in the Netherlands and, apart from the patient described above, a further two patients with iatrogenic CJD have been identified, who developed the disease after dura mater transplantation. ${ }^{11}$

An incubation period as long as 38 years had never been reported for iatrogenic CJD. Huillard d'Aignaux et al studied the incubation period in 55 patients with hGH related CJD in a cohort of 1361 French hGH recipients. The median incubation period was between 9 and 10 years. Under the most pessimistic model, the upper limit of the $95 \%$ confidence interval varied between 17 and 20 years. Although the infecting dose cannot be quantified, it can be speculated that the long incubation period in our patient is partly explained by the administration of a limited amount of hGH. This hypothesis is supported by experimental models, in which higher infecting doses usually produce shorter incubation periods. ${ }^{6}$ Since our patient was one of the first in the world to receive hGH, this case indicates that still more patients with iatrogenic CJD can be expected in the coming years. Another implication of our study is that CJD can develop even after a low dose of hGH. This case once more testifies that worldwide close monitoring of any form of iatrogenic CJD is mandatory.

\section{ACKNOWLEDGEMENTS}

We are grateful to M Jansen PhD MD for his search for the origin of the growth hormone and P P Taminiau MD. CJD surveillance in the Netherlands is carried out as part of the EU Concerted Action on the Epidemiology of CJD and the the EU Concerted Action on Neuropathology of CJD, both funded through the BIOMED II programme, and is supported by the Dutch Ministry of Health. This surveillance would not have been possible without the cooperation of all Dutch neurologists and geriatricians.

\section{Authors' affiliations}

E A Croes, G Roks*', C M van Duijn, Genetic Epidemiology Unit, Department of Epidemiology and Biostatistics, Erasmus University Medical Centre Rotterdam, PO Box 1738, 3000 DR Rotterdam, Netherlands

P C G Nijssen, Department of Neurology, St Elisabeth Hospital, PO Box 90151, 5000 LC Tilburg, Netherlands

G H Jansen, Department of Pathology, University Medical Centre Utrecht, Heidelberglaan 100, 3584 CX Utrecht, Netherlands

*Also the Department of Neurology, St Elisabeth Hospital

Correspondence to: Professor C M van Duijn, Genetic Epidemiology Unit, Department of Epidemiology and Biostatistics, Erasmus University Medical Centre Rotterdam, PO Box 1738, 3000 DR Rotterdam,

Netherlands; vanduijn@epib.fgg.eur.n

Received 27 December 2001

In revised form 1 March 2002

Accepted 12 March 2002

Competing interests: none declared

\section{REFERENCES}

1 Duffy P, Wolf J, Collins $G$, et al. Possible person-to-person transmission of Creutzfeldt-Jakob disease. N Engl J Med 1974;290:692-3.

2 Bernoulli C, Siegfried J, Baumgartner G, et al. Danger of accidental person-to-person transmission of Creutzfeldt-Jakob disease by surgery. Lancet 1977:i:478-9.

3 Koch TK, Berg BO, De Armond SJ, et al. Creutzfeldt-Jakob disease in a young adult with idiopathic hypopituitarism: possible relation to the administration of cadaveric human growth hormone. N Engl J Med 1985;313:731-3.

4 Cochius JI, Burns RJ, Blumbergs PC, et al. Creutzfeldt-Jakob disease in a recipient of human pituitary-derived gonadotrophin. Aust NZ J Med 1990;20:592-3.

5 Thadani V, Penar PL, Partington J, et al. Creutzfeldt-Jakob disease probably acquired from a cadaveric dura mater graft: case report. J Neurosurg 1988:69:766-9.

6 Brown P, Preece M, Brandel JP, et al. latrogenic Creutzfeldt-Jakob disease at the millennium. Neurology 2000;55:1075-81.

7 Huillard d'Aignaux J, Costagliola D, Maccario J, et al. Incubation period of Creutzfeldt-Jakob disease in human growth hormone recipients in France. Neurology 1999;53:1 197-201.

8 Billette de Villemeur T, Deslys JP, Pradel A, et al. Creutzfeldt-Jakob disease from contaminated growth hormone extracts in France. Neurology 1996;47:690-5

9 Roos RA, Wintzen AR, Will RG, et al. Een patiënt met de ziekte van Creutzfeldt-Jakob na behandeling met humaan groeihormoon. Ned Tiidschr Geneeskd 1996;140:1 190-3.

10 Wientiens DP, Rikken B, Wit JM, et al. A nationwide cohort study on Creutzfeldt-Jakob disease among human growth hormone recipients. Neuroepidemiology 2000;19:201-5.

11 Croes EA, Jansen GH, Lemstra AF, et al. The first two patients with dura mater associated Creutzfeldt-Jakob disease in the Netherlands. J Neurol 2001;248:877-81 